\title{
PRESENCIA DEL GÉNERO TROPIDOPHIS (SERPENTES: TROPIDOPHIIDAE) EN EL SISTEMA NACIONAL DE ÁREAS PROTEGIDAS DE CUBA
}

\section{PRESENCE OF THE GENUS TROPIDOPHIS (SERPENTES: TROPIDOPHIDAE) IN THE CUBAN NATIONAL SYSTEM OF PROTECTED AREAS}

\author{
RODRÍGUEZ-S, LOURDES, ${ }^{1 *}$ Dra., RIVALTA-G, VILMA, ${ }^{1}$ M.Sc., \\ GONZÁLEZ-R, AMNERYS, ${ }^{2 * *}$ M.Sc, FERNÁNDEZ DE ARCILA-F, ROLANDO,${ }^{2}$ Lic.
}

${ }^{1}$ Instituto de Ecología y Sistemática, Carretera de Varona 11835, e/ Oriente y Lindero, Boyeros, La Habana 19, CP 11900, Cuba. ${ }^{2}$ Centro Nacional de Áreas Protegidas, Calle 18A4114, e/ 41 y 47, Playa, La Habana 13, CP11300, Cuba.

Palabras Clave:

Áreas protegidas, Boas enanas, Distribución geográfica, Conservación, Cuba.

Key words:

Protected areas, Dwarf boas, Geographic distribution, Conservation, Cuba.

\section{Resumen}

Del género Tropidophis Cocteau y Bibron, 1843, en la subregión antillana existen 27 especies, de las cuales 16 son cubanas, todas endémicas. El Sistema Nacional de Áreas Protegidas en Cuba (SNAP) comprende 211 áreas protegidas, caracterizadas en el Plan del Sistema Período de 2014-2020. El objetivo de este trabajo es verificar cuáles especies del género Tropidophis se encuentran cubiertas porelSNAP, comounadelas vías para su consenvación. Se revisólaliteratura para determinarla distribución geográfica, así comose chequearon los ejemplares de colecciones cubanas y del mundo, con el mismo fin. Cada localidad fue georeferenciada e incluida como una capa en el Sistema de Información Geográica Mapinfo 6.5, el cual contiene una capa con los limites actualizados de lasáreas protegidas. Sobreestainformación se determinóqueunaespecie está cubierta por el SNAP si, al menos, una de sus localidades se encuentra en alguna de las 211 áreas protegidas. Seencontróque solo dos especies no cumplen con este precepto. Debidoaque algunas especies son locales y otras tienen distribución geográfica limitada a pocas localidades, todas ellas están incluidas en el Apéndice II de la CITES y en el Apéndice I de la Resolución 160 de 2011 del Ministro de Ciencia, Tecnología y Medio Ambiente y 11 fueron categorizadas en el Libro Rojo de los Vertebrados de Cuba. Por tanto, estas especies se deben tener en cuenta en los planes de manejo y conservación de las áreas protegidas de Cuba.

\section{Abstract}

In the West Indies there are 27 species of the genus Tropidophis Cocteau and Bibron, 1843, 16 of them are endemic to Cuba. The Cuban National System of Protected Areas (CNSPA) encompasses 211 protected areas, described in the System Plan Period of 2014-2020. The main goal of this work is to verify what species of the genus Tropidophis are present in the CNSPA, as a one of the ways for its conservation. We revised the literature on geographic distribution, also the data bases of several Cuban and worldwide collections. For every found locality was determined its geographic coordinates and included in a Geographic Information System Mapifo 6.5 which has a sheet with the up to date limits of the protected areas. We assumed that a species is protected for the NSPA if, at least, one of its localities is included in one of the 211 protected areas. We found that just two species are out the system. Because some of the species are locally distributed, they are in the Appendix II of CITES, in the Appendix I of the Resolution 160 of the Minister of Science, Technology and Environment and 11 species were categorized as threatened in the Red Book of the Cuban Vertebrates. That is why the species of this genus must be included in the management and conservation plans in the protected areas of Cuba. 


\section{Introducción}

Las boas enanas pertenecen a la familia Tropidophiidae Brongersma, 1951, que está compuesta por dos géneros: Trachyboa Peters, 1860, con distribución centroamericana, y Tropidophis Cocteau y Bibron, 1843, con distribución Neotropical (VIDAL y HEDGES, 2009). Este último género cuenta, hasta el presente, con 30 especies, de las cuales tres pertenecen a Suramérica y el resto a las Antillas (HEDGES, 2002; HENDERSON y POWELL, 2009). Cuba tiene el mayor número de especies (16), todas endémicas (DOMÍNGUEZ et al., 2006; HENDERSON y POWELL, 2009), por lo cual es el centro de diversidad del género dentro del área geográfica antillana. De manera general, son serpientes de pequeño tamaño, patrón de colores típicamente manchado, vivíparas, casi siempre de hábitos nocturnos y terrestres, son inofensivas totalmente y se alimentan de pequeños vertebrados (HENDERSON y POWELL, 2009).

Las especies cubanas son: Tropidophis celiae Hedges, Estrada y Díaz, 1999 (Boca de Canasí y Carbonera); T. feicki Schwartz, 1957 (Cordillera de Guaniguanico y Ciénaga de Zapata); T. fuscus Hedges y Garrido, 1992 (norte oriental); T. galacelidus Schwartz y Garrido, 1975 (sur de Cienfuegos y de Sancti Spíritus); T. hardyi Schwartz y Garrido, 1975 (sur de Cienfuegos y de Sancti Spíritus); T. hendersoni Hedges y Garrido, 2002 (Guardalavaca y Gibara); T. maculatus (Bibron, 1840, en Cocteau y Bibron, 1843) (Cuba occidental e Isla de la Juventud); T. melanurus (Schlegel, 1837) (Cuba e Isla de la Juventud); T. morenoi Hedges, Garrido y Díaz, 2001 (Caguanes y Loma de Cunagua); T. nigriventris Bailey, 1937 (Camagüey oriental); T. pardalis (Gundlach, 1840) (Cuba e Isla de la Juventud); T. pilsbryi Bailey, 1937 (Cuba oriental); T. semicinctus (Gundlach y Peters, 1865) (Cuba occidental y central); T. spiritus Hedges y Garrido, 1999 (centro sur de Cuba); T. wrighti Stull, 1928 (centro y oriente de Cuba); T. xanthogaster Domínguez, Moreno y Hedges, 2006 (Guanahacabibes). Todas están incluidas en el Apéndice II de la CITES y en el I de la Resolución 160 de 2011 del Ministerio de Ciencia, Tecnología y Medio Ambiente de Cuba. Sin embargo, en el Libro Rojo de los Vertebrados de Cuba (González Alonso et al., 2012) solo se incluyeron las más amenazadas: T. celiae (Fig. 1), T. feicki, T. fuscus, T. galacelidus, T. hardyi, T. hendersoni, T. morenoi (Fig. 2), T. nigriventris, T. pilsbryi, T. spiritus (Fig. 3) y T. xanthogaster.

La información sobre las especies de este género se encuentra dispersa en numerosas publicaciones seriadas y libros. Por ello, el objetivo de este trabajo es compilar los datos sobre la distribución geográfica y relacionar esta con las áreas protegidas del país, para verificar cuáles especies del género Tropidophis se encuentran cubiertas por el Sistema Nacional de

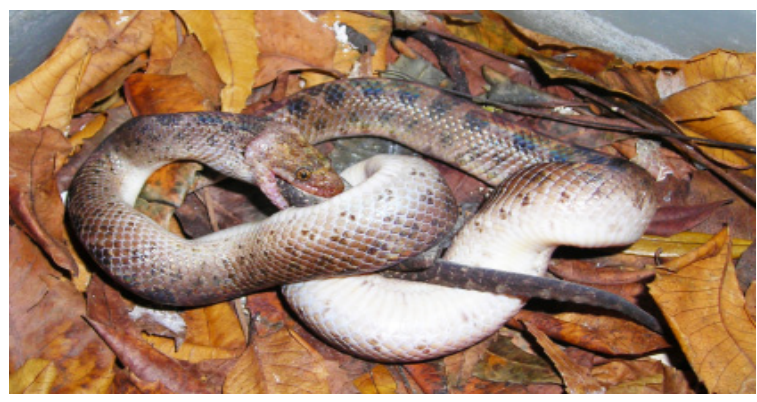

Figura 1. T.celiae

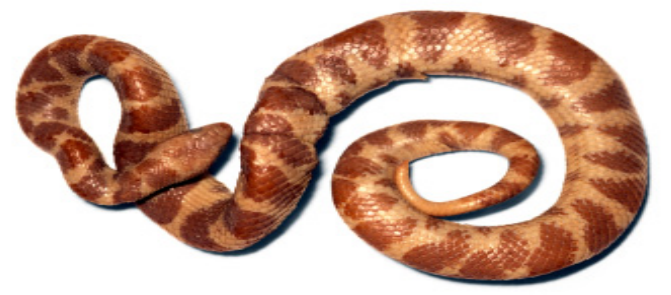

Figura 2. T. morenoi

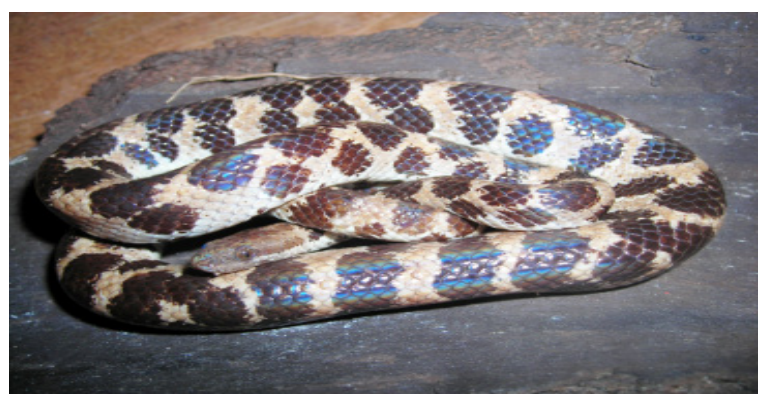

Figura 3. T. spiritus

Fuente: Libro Rojo de los Vertebrados de Cuba (González Alonso, H., L. Rodríguez Schettino, A. Rodríguez, C. A. Mancina e I. Ramos García, Eds.)

Áreas Protegidas de Cuba (SNAP), como una de las vías para su conservación.

\section{Materiales y métodos}

Se revisó la literatura sobre el género, hasta el 2014, y se examinaron las bases de datos de colecciones de Cuba y del mundo: CZACC (Instituto de Ecología y Sistemática, La Habana); MNHNCu (Museo Nacional de Historia Natural de Cuba, La Habana); MCG (Museo Cubano Gundlach, La Habana); BIOECO (Centro Oriental de Ecosistemas y Biodiversidad, Santiago de Cuba); CIEC (Centro de Investigaciones de Ecosistemas Costeros, Ciego de Ávila); ECOVIDA (Museo de Historia Natural Tranquilino Sandalio de Noda, Pinar del Río); USNM (National Museum of Natural History, Washington, DC); MCZ (Museum of Comparative Zoology, Harvard University); AMNH 
(American Museum of Natural History, New York); CASSU (California Academy of Science, San Francisco); ANSP (Academy of Natural Science, Philadelphia); CM (Carnegie Museum of Natural History, Pittsburgh);FMNH (Field Museum of Natural History, Chicago); FM (Florida Museum of Natural History, University of Florida); KU (University of Kansas Natural History Museum); LACM (Natural History Museum, Los Angeles County); LSUMZ (Museum of Zoology, Louisana State University); MVZ (Museum of Vertebrate Zoology, University of California, Berkeley); UMMZ (Museum of Zoology, University of Michigan); ROM (Royal Ontario Museum, Canada); ZMB (Museumfür Naturkunde, Humboldt Universität, Berlin).

Las localidades encontradas fueron georeferenciadas e incluidas en una capa en el Sistema de Información Geográfica Mapinfo 6.5, el cual contiene una capa con los límites actualizados de las áreas protegidas (CNAP, 2013).

Para determinar si una especie se encuentra cubierta por el SNAP se valoró si, al menos, una de sus localidades queda dentro de los límites de alguna de las 211 áreas protegidas.

\section{Resultados y discusión}

El Sistema Nacional de Áreas Protegidas de Cuba (SNAP) comprende 211 áreas caracterizadas en el Plan del SNAP Período de 2014-2020 y cubren $20,20 \%$ del territorio nacional. De significación nacional (APSN) son 77 y 134 son de significación local (APSL) (Martínez et al., 2013). Las áreas protegidas (AP) están clasificadas en ocho categorías de manejo (Tabla 1). Las provincias con mayor número de AP son Camagüey, Guantánamo y Pinar del Río; con menos números, Mayabeque, Cienfuegos y Las Tunas.

Tabla 1. Número de áreas protegidas por categoría de manejo y por nivel de Significación.

\begin{tabular}{lccc}
\hline \multirow{2}{*}{ Categoría de Manejo } & \multicolumn{3}{c}{ No. de áreas protegidas } \\
\cline { 2 - 4 } & APSN & APSL & Total \\
\hline Reserva Nacional (RN) & 4 & 0 & 4 \\
Parque Nacional (PN) & 14 & 0 & 14 \\
Reserva Ecológica (RE) & 19 & 13 & 32 \\
Elemento Natural Destacado (END) & 10 & 23 & 33 \\
Reserva Florística Manejada (RFM) & 7 & 34 & 41 \\
Refugio de Fauna (RF) & 11 & 34 & 45 \\
Paisaje Natural Protegido (PNP) & 2 & 22 & 24 \\
Área Protegida de Recursos Manejados (APRM) & 10 & 8 & 18 \\
\hline $\begin{array}{l}\text { APSN, área protegida de significación Nacional; } \\
\text { significación local (MARTíNEZ et al., 2013) }\end{array}$ & \multicolumn{3}{c}{ (10 protegida de }
\end{tabular}

Las amenazas principales para este género son la fragmentación o pérdida del hábitat por agricultura, urbanización y desarrollo socioeconómico en general; las especies invasoras como la mangosta, el perro, el gato y el puerco jíbaros; los mitos: miedo, aversión; el efecto del cambio climático (aumento de la temperatura ambiental, variación en el patrón de las precipitaciones, sequías, elevación del nivel medio del mar) (HENDERSON y POWELL, 2009; GONZÁLEZ ALONSO et al., 2012). Las especies con distribución geográfica restringida y ecológica limitada a pocos parches del hábitat y las que viven muy cerca de las costas son las que tienden a estar más amenazadas.

Las vías para su conservación son in situ, ex situ o ambas, además de otras medidas como la educación y la legislación ambiental (RODRÍGUEZ SCHETTINO et al., 2012). La principal vía in situ es mediante el establecimiento de áreas protegidas. Las especies de este género se han encontrado, hasta el presente, en 31 APSN e igual número de APSN, lo que es el $29,4 \%$ del total de áreas protegidas del SNAP.

Las especies con mayor cobertura son T. melanurus, $T$. pardalis y $T$. semicinctus, que son las de distribución geográfica más amplia y no fueron categorizadas como amenazadas (Tabla 2). Otras dos no amenazadas ( $T$. maculatus y $T$. wrighti) están presentes en ocho AP cada una. Sin embargo, las 11 especies amenazadas están presentes entre 1 y 6 AP y de ellas, dos no están cubiertas por el SNAP.

Tres especies (T. celiae, T. galacelidus y $T$. spiritus) están cubiertas por un AP (Tabla 2). La primera en Boca de Canasí, la segunda en Topes de Collantes y la tercera en Lomas de Banao (Tabla 3). TORRES et al. (2013) añadieron la localidad de Carbonera, provincia de Matanzas para T. celiae, que queda entre dos AP, Laguna de Maya y Cueva de Santa Catalina. RIVALTA et al. (2013) mencionaron cinco ejemplares de Banao para T. galacelidus depositados en la colección herpetológica del IES, por lo que pudiera estar presente en la Reserva Ecológica Lomas de Banao. FONG y DE ARMAS (2011) registraron $T$. spiritus en la loma del Heliógrafo, cerca de la Reserva Florística Manejada Lebrije.

Una de las tres localidades conocidas de T. hardyi está muy cerca del PNP Topes de Collantes y las de $T$. hendersoni se hallan próximas al PNP Bahía de Naranjo (RODRÍGUEZ SCHETTINO et al., 2013; GONZÁLEZ et al., 2013) y a la RE Caletones y al RF Balsas de Gibara, de acuerdo con DÍAZ et al. (2014) que amplían la distribución de esta especie a la ciudad de Gibara. Por ello, un estudio más preciso podría añadir más localidades a las tres especies presentes en una sola AP y a las dos que no están cubiertas por el SNAP. 
Tabla 2. Categorías de amenaza y número de AP por especies del género Tropidophis en Cuba.

\begin{tabular}{|c|c|c|}
\hline Especies & $\begin{array}{l}\text { Categoría de amenaza } \\
\text { (GONZÁLEZ et al. 2012) }\end{array}$ & No. de áreas protegidas \\
\hline T. celiae & $C R$ & 1 \\
\hline T. feicki & VU & 5 \\
\hline T. fuscus & $C R$ & 3 \\
\hline T. galacelidus & $C R$ & 1 \\
\hline T. hardyi & CR & 0 \\
\hline T. hendersoni & $\mathrm{CR}$ & 0 \\
\hline T. maculatus & -- & 8 \\
\hline T. melanurus & -- & 53 \\
\hline T. morenoi & $\mathrm{CR}$ & 4 \\
\hline T. nigriventris & $\mathrm{CR}$ & 2 \\
\hline T. pardalis & -- & 25 \\
\hline T. pilsbryi & $\mathrm{CR}$ & 4 \\
\hline T. semicinctus & - & 14 \\
\hline T. spiritus & $\mathrm{CR}$ & 1 \\
\hline T. wrighti & -- & 8 \\
\hline T. xanthogaster & EN & 2 \\
\hline
\end{tabular}

Estos estudios se podrían realizar hacia el sur de la provincia de Cienfuegos para T. hardyi, ya que la distribución de esta especie se encuentra entre Soledad y Trinidad, por lo que se pudiera hallar en las áreas protegidas de Guanaroca-Punta Gavilán, Guajimico y Topes de Collantes (GONZÁLEZ et al., 2013). De igual forma, se deberá acometer una búsqueda más precisa en los alrededores del área de distribución de T. hendersoni, en la costa norte de Holguín.

Las 16 especies se pueden clasificar en cuatro grupos, según el número de AP y los registros de presencia: 1más de 10 AP (3 especies); 2- de 2 a 9 AP (8 especies); 3- con un AP (3 especies); 4-no presentes en AP (2 especies) (Tablas 2 y 3 ). El género Tropidophis en Cuba tiene buena cobertura por el SNAP, ya que 14 de 16 especies $(87,5 \%)$ se hallan en, al menos, un AP, lo que es una vía para su protección y conservación.

De las 11 especies con categoría de amenaza, 9 $(81,8 \%)$, tienen registros de distribución en las AP del SNAP con diferentes categorías de manejo y casi todas disponen de administración (Tabla 3, Fig. 4).

Dado su atractivo, estas especies pudieran ser objeto de comercio, por lo que a nivel internacional todas las especies del género se encuentran incluidas en el Apéndice II de la Convención sobre el comercio internacional de especies amenazadas de fauna y flora silvestres (CITES), donde figuran las especies que no están necesariamente amenazadas de extinción pero que podrían llegar a estarlo, a menos que se controle estrictamente su comercio.
Tabla 3. Especies del género Tropidophis con categoría de amenaza y áreas protegidas donde se encuentran presentes.

\begin{tabular}{|c|c|c|c|}
\hline Especie & $\mathrm{CA}^{*}$ & AP & Admón \\
\hline \multirow{6}{*}{$\begin{array}{l}\text { Tropidophis celiae } \\
\text { Tropidophis feicki }\end{array}$} & \multirow[t]{3}{*}{$\mathrm{CR}$} & RFM Boca de Canasí & no \\
\hline & & APRM RB Sierra del Rosario & si \\
\hline & & APRM Península de Zapata & si \\
\hline & \multirow[t]{3}{*}{ VU } & PN Viñales & si \\
\hline & & RFM San Ubaldo-Sabanalamar & si \\
\hline & & END Pan de Guajaibón & si \\
\hline \multirow[t]{3}{*}{ Tropidophis fuscus } & & PN Alejandro de Humboldt & si \\
\hline & \multirow[t]{2}{*}{$\mathrm{CR}$} & APRM Cuchillas del Toa & si \\
\hline & & END Yunque de Baracoa & si \\
\hline \multirow{4}{*}{$\begin{array}{l}\text { Tropidophis galacelidus } \\
\text { Tropidophis hardyi } \\
\text { Tropidophis hendersoni } \\
\text { Tropidophis morenoi }\end{array}$} & $\mathrm{CR}$ & PNP Topes de Collantes & si \\
\hline & CR & - & - \\
\hline & \multirow[t]{2}{*}{$\mathrm{CR}$} & - & - \\
\hline & & PN Caguanes & si \\
\hline \multirow{5}{*}{ Tropidophis nigriventris } & \multirow{3}{*}{ CR } & RF Loma de Cunagua & si \\
\hline & & APRM Buenavista & si \\
\hline & & RE Centro y Oeste de Cayo Coco & si \\
\hline & \multirow{2}{*}{$\mathrm{CR}$} & APRM Sierra del Chorrillo & si \\
\hline & & PNP Sierra de Najasa & no \\
\hline \multirow[t]{4}{*}{ Tropidophis pilsbryi } & & PN Pico Bayamesa & si \\
\hline & \multirow{3}{*}{$\mathrm{CR}$} & PNP Gran Piedra & si \\
\hline & & APRM Baconao & si \\
\hline & & PN La Mensura-Pilotos & si \\
\hline \multirow{3}{*}{$\begin{array}{l}\text { Tropidophis spiritus } \\
\text { Tropidophis xanthogaster }\end{array}$} & $\mathrm{CR}$ & RE Lomas de Banao & si \\
\hline & & APRM Península de Guanahacabibes & si \\
\hline & EIV & PN Guanahacabibes & si \\
\hline
\end{tabular}

CA*: Categoría de amenaza (GONZÁLEZ et al. 2012)

Por otra parte, en la Lista Roja de la Unión Internacional para la Conservación de la Naturaleza (IUCN, 2014) se encuentran solamente evaluadas $T$. hendersoni (CR) y T. pardalis (LC). La Alianza para la Extinción Cero (17 sitios identificados en Cuba), no incluyen este género, no obstante, en ellos se pueden encontrar estas especies, según su distribución geográfica.

En nuestro país, la Resolución No. 160 /2011 del CITMA establece las regulaciones para el control y la protección de especies de especial significación para la diversidad biológica, la cual define las listas de especies, ya sea por su endemismo, por ser amenazadas o en peligro de extinción, por su representatividad en los ecosistemas, por sus elevados valores ecológicos, económicos o de otra índole, o por estar protegidas por los convenios internacionales de los que Cuba es Estado Parte; así como para controlar la utilización sostenible de dichas especies y su exportación. En esta resolución se consideran todas las especies del género Tropidophis dentro del Apéndice I, lo que refuerza las medidas internacionales para su conservación. Además, en el Libro Rojo de los Vertebrados de Cuba (2012) se evaluaron 11 especies: 9 En Peligro Crítico, 1 En Peligro y 1 Vulnerable. 


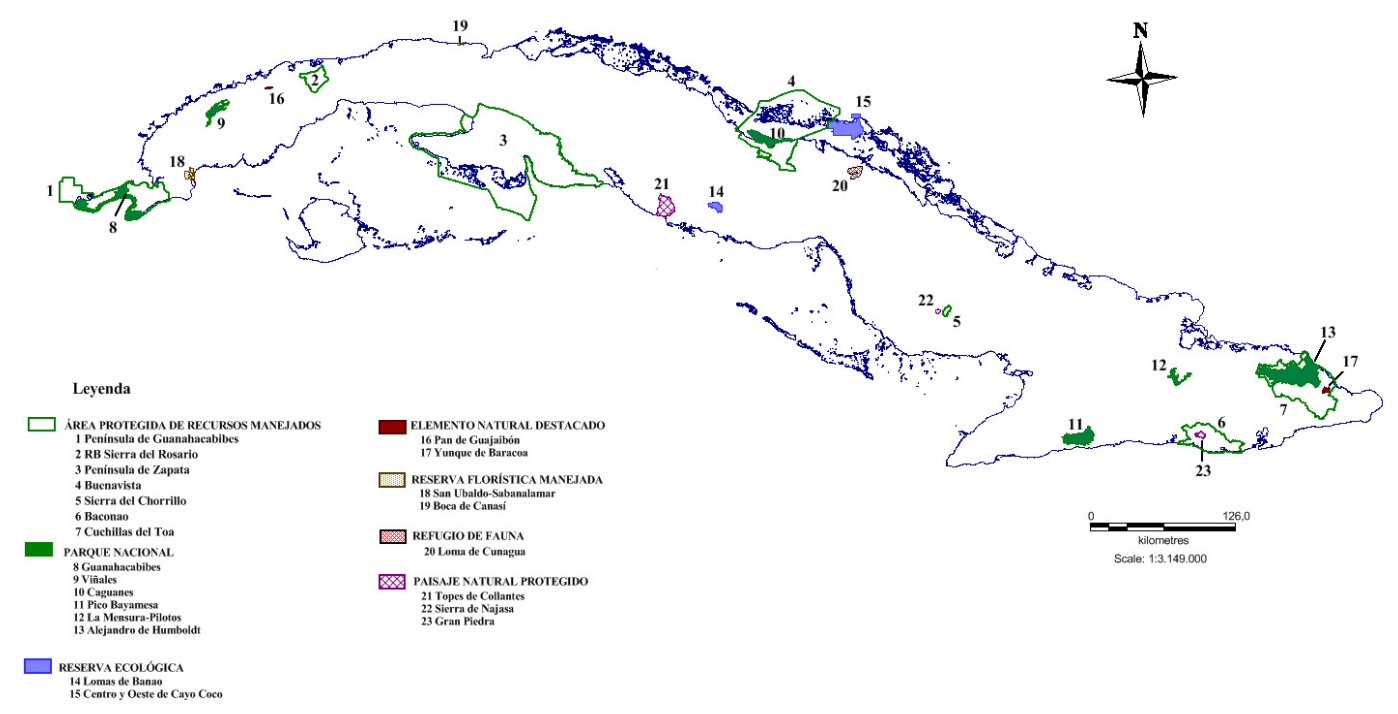

Figura 4. Localización geográfica de las áreas protegidas donde está presente el género Tropidiphis en Cuba

Otras medidas encaminadas a preservar estas especies se relacionan con la conservación ex situ, como la cría en cautiverio en parques zoológicos. Por otra parte, existe la cría en cautiverio por personas particulares, que debe estar debidamente registrada ante las autoridades regulatorias competentes.

La principal herramienta de las áreas protegidas administradas es su plan de manejo, con el que se planifican las acciones que se ejecutan mediante los planes operativos anuales, a través de diferentes programas de manejo, entre ellos los de investigación y monitoreo. A estos planes se les podrían incorporar labores encaminadas a profundizar en el estudio de estas especies, en colaboración con expertos e instituciones dedicadas a las investigaciones, por lo que deben ser tenidos en consideración a los efectos de la planificación de nuevas áreas protegidas. En estos planes también se incluyen programas de educación ambiental que involucran a las comunidades locales insertas o aledañas a las áreas, lo que también puede ayudar a incrementar el conocimiento sobre la importancia y necesidad de conservar estas y otras especies, pero se debe hacer mayor esfuerzo encaminado especialmente a los ofidios, en general. Igualmente, este tipo de educación puede hacerse extensivo a la población cubana, mediante la divulgación utilizando medios de comunicación masiva.

También es importante gestionar la administración de las áreas protegidas RFM Boca de Canasí y PNP Sierra de Najasa por parte de las autoridades ambientales provinciales, para poder brindar una mayor protección a los recursos naturales que estos sitios poseen.

Asimismo, los que toman decisiones sobre licencias ambientales y estudios de impacto ambiental deben tener en cuenta la información sobre este género expuesta en este trabajo.

Agradecimientos: A Manuel Iturriaga por brindar literatura y por revisar el manuscrito.

\section{Referencias}

CNAP. 2013. Plan del Sistema Nacional de Áreas Protegidas de Cuba. Período 2014-2020. Ministerio de Ciencia, Tecnología y Medio Ambiente, La Habana, Cuba.

DÍAZ, L. M.; CÁDIZ, A.; VILLAR, S.; BERMÚDEZ, F. 2014. Notes on the ecology and morphology of the Cuban khaki trope, Tropidophis hendersoni Hedges and Garrido (Squamata: Tropidophiidae), with a new locality record. IRCF Reptiles \& Amphibians 21(4):116-119. 
DOMÍNGUEZ DÍAZ, M.; MORENO, L. V.; HEDGES, S. B. 2006. A new snake of the genus Tropidophis (Tropidophiidae) from the Guanahacabibes Peninsula of Western Cuba. Amphibia-Reptilia 27:427-432.

FONG G., A.; ARMAS, L. F. DE. 2011. The easternmost record for Tropidophis spiritus Hedges and Garrido, 1999 (Serpentes: Tropidophiidae) in Cuba. Herpetology Notes 4:111-112.

GONZÁlEZ ALONSO, H.; RODRÍGUEZ SCHETTINO, L.; RODRÍGUEZ, A.; MANCINA, C. A.; RAMOS GARCÍA, I. 2012. Libro Rojo de los Vertebrados de Cuba. Editorial Academia. Cuba.

GONZÁlEZ, A.; FERNÁNDEZ DE ARCILA, R.; AGUILAR, S. 2013. Metas de conservación y análisis de vacío. Vertebrados. En Plan del Sistema Nacional de Áreas Protegidas de Cuba. Período 2014-2020. Ministerio de Ciencia, Tecnología y Medio Ambiente. La Habana, Cuba.

HEDGES, S. B. 2002. Morphological variation and the definition of species in the snake genus Tropidophis (Serpentes, Tropidophiidae). Bulletin of the Natural History Museum of London (Zoology) 68(2):83-90.

HEDGES, S. B.; GARRIDO, O. H. 2002. A new snake of the genus Tropidophis (Tropidophiidae) from eastern Cuba. Journal of Herpetology 36(2):157-161.

HENDERSON, R. W.; POWELL, R. 2009. Natural History of West Indian Reptiles and Amphibians. Univ. Press Florida. Gainesville, USA.

IUCN. 2014. Red List of Threatened Species, v. 3.Disponible en: http://www.iucnredlist.org/. Consultado: 10-12-2014.

MARTínEZ, A. J.; VALDÉS, J. A.; LORENZO, C.; HERNÁNDEZ, A.; PERERA, S.; DÍAZ, C.; QUEVEDO, O. 2013. Distribución espacial. En Plan del Sistema Nacional de Áreas Protegidas de Cuba. Período 2014-2020. Ministerio de Ciencia, Tecnología y Medio Ambiente. La Habana, Cuba.

RIVALTA GONZÁLEZ, V.; GONÁLEZ CARRALERO, A.; RODRÍGUEZ SCHETTINO, L. 2013. Colección herpetológica del Instituto de Ecología y Sistemática, La Habana, Cuba. Familia Tropidophiidae, género Tropidophis. Revista Colombiana de Ciencia Animal 5(2):282-300.

RODRÍGUEZ SCHETTINO, L.; MANCINA C. A.; RIVALTA GONZÁLEZ, V. 2013. Reptiles of Cuba: Checklist and Geographic Distributions. Smithsonian Herpetological Information Service 144:1-92.

RODRÍGUEZ SCHETTINO, L.; RIVALTA GONZÁLEZ, V.; PREDE RODRÍGUEZ, M. L; REGALADO CALERO, T. 2012. Vías para la conservación de la diversidad biológica. Diversidad Biológica de Cuba. Cuba.

TORRES LÓPEZ, J.; TORRES FUNDORA, O.; MARRERO, R. 2013. Nueva localidad para la boa enana Tropidophis celiae Hedges, Estrada y Díaz, 1999 Serpentes, Tropidophiidae). Revista Cubana de Ciencias Biológicas 2(3):79-82.

VIDAL, N.; HEDGES, S. B. 2009. The molecular evolutionary tree of lizard, snakes, and amphisbaenians. C. R. Biologies 332:129-139. 